\title{
LA CALIDAD DE LA EDUCACIÓN EN LA SOCIEDAD DEL CONOCIMIENTO
}

\author{
Gabriela Iturralde Espejo
}

\section{RESUMEN}

La globalización, y todo lo que ésta implica, ha generado gran cantidad de cambios en las formas de ser, pensar y actuar de las personas y las organizaciones. En la actualidad, el conocimiento proporciona el valor agregado, sin embargo debe acompañarse por una serie de actitudes que permitan actuar de forma responsable y comprometida. La principal fuente de conocimientos y actitudes es la educación. De ahí que, en los últimos años, los esfuerzos en materia educativa han sido encaminados a mejorar la calidad de la misma en todos sus niveles.

A nivel Proceso Enseñanza Aprendizaje (PEA), la calidad se logra cuando: la educación es completa, cumple los objetivos que se plantea y existe coherencia entre los elementos que la conforman, dando respuesta a las demandas de la comunidad en que se lleva a cabo.

A nivel nacional, se alcanza la calidad cuando: se logran los objetivos planteados por el sistema, se satisfacen las necesidades de la sociedad, hay igualdad de oportunidades entre los diferentes sectores de la población, se emplean convenientemente los recursos y existe una adecuada administración de políticas; de este modo se promueve el desarrollo económico, político y social de la nación.

Por su parte, el nivel internacional señala que la educación es de calidad cuando: el alumno aprende a aprender, desarrolla competencias, es capaz de relacionarse con otros y desarrolla su propia personalidad. Contar con una educación de calidad uniforme en todas las naciones, genera un desarrollo equitativo a nivel mundial. 
La calidad en todos los niveles es un proceso compuesto por varios pasos: diagnóstico, planeación, implementación y análisis de evidencias. Es un proceso cíclico; cada evidencia representa un nuevo diagnóstico. La calidad sugiere un proceso de mejora continua; nunca se alcanza la calidad total.

\section{INTRODUCCIÓN}

El objetivo principal de este ensayo es analizar lo que se entiende por calidad de educación, desde los diferentes niveles de organización educativa, en el contexto de la sociedad del conocimiento. La hipótesis que se quiere desarrollar es la siguiente: una educación de calidad es aquella que responde a las necesidades de cada región, dadas por sus características culturales, políticas, geográficas, demográficas, económicas, etcétera, y al mismo tiempo atiende a las necesidades del mundo global en que se desenvuelve. La educación, para ser considerada de calidad, debe responder a las características del tiempo y lugar en que se ubica, de tal modo que promueva el desarrollo individual y común.

No puede hablarse de educación de calidad si primero no se comprende lo que es la educación; de ahí que comenzaremos definiendo la educación y sus funciones. En un primer momento, para ser de calidad, la educación debe cumplir con el fin que se le ha encomendado.

El concepto de calidad en la educación puede ser analizado en los diversos niveles de la organización educativa, desde la relación educando-educador hasta las políticas educativas internacionales, pasando por el aula, las instituciones y los sistemas educativos nacionales. Para tener una visión más completa del término, lo abordaremos haciendo un breve análisis del concepto y los parámetros que se tienen en cada nivel.

El primer nivel será el Proceso Enseñanza Aprendizaje como tal; analizaremos la calidad en cuanto al hecho educativo (relación educando-educador, objetivos, contenido, metodología, recursos, evaluación, comunidad educativa, familia, etcétera) y en lo relativo a la gestión escolar. 
Posteriormente, reflexionaremos sobre el concepto de calidad a nivel de los sistemas educativos nacionales. Para ello, tomaremos como base los parámetros utilizados por varios autores para analizar dichos sistemas.

La última categoría de análisis será la calidad de la educación a nivel internacional; nos basaremos en los pilares de la educación dados por la UNESCO (Organización de las Naciones Unidas para la Educación, la Ciencia y la Cultura), y en los requisitos que deben cumplir los programas para ser considerados de calidad, propuestos por la UNESCO en el Foro Mundial de Educación (Dakar, 2000).

Durante el análisis de este tema, nos percataremos que para alcanzar una verdadera educación de calidad se exige un trabajo interdisciplinario que permita tener una visión de conjunto.

Finalmente, realizaremos una comparación entre las exigencias de la sociedad del conocimiento y las características de una educación de calidad, antes analizadas.

Terminaremos este ensayo proporcionando las conclusiones de la hipótesis planteada al inicio. Utilizaremos un esquema que sintetiza de forma gráfica los elementos que intervienen en la calidad de la educación.

\section{LA EDUCACIÓN Y SUS FUNCIONES}

Por la raíz etimológica de la palabra educación -educere y educare-, se infiere que se trata de un proceso en donde se extrae o saca y conducen las potencialidades de la persona para promover su desarrollo.

Entendida así, la educación se enfatiza como el proceso de perfeccionamiento de las capacidades propias de la persona, en todos sus ámbitos (físico, psicológico, intelectual, espiritual y social) y durante todas las etapas de su vida; que promueve el desarrollo individual y común, y representa un hecho social indispensable para el desarrollo de las colectividades. 
Si bien es cierto que la educación es eminentemente un hecho individual, tiene también carácter social; no puede concebirse al individuo aislado, ni a la educación sin contar con un ser que aprenda y otro que enseñe o muestre. Como dice Durkheim, la educación es, por lo tanto, la acción ejercida por las generaciones adultas sobre las jóvenes, que permite la relación intergeneracional y la reproducción del grupo.

De ahí que especialistas de la UNESCO, en el libro Educación: la agenda hacia el siglo XXI, hacia un desarrollo humano, clasifiquen las funciones de la educación de la siguiente manera:

a) En términos individuales, es la preparación para asumir determinados papeles. Se pueden dividir en tres: la función de socializar, transmitir la cultura y desarrollar la personalidad; formar para el trabajo; y entrenar para la ciencia y la tecnología.

b) En términos sociales, se trata de la circulación del conocimiento, y hace referencia a los objetivos sociales que suelen atribuirse a la educación: integración nacional, crecimiento económico y superación de la pobreza ${ }^{1}$.

A continuación, analizaremos brevemente cada una de estas funciones, por ser indispensables para una educación de calidad.

La principal tarea de la educación es preparar para la vida (futuro) y durante la vida (presente), lo cual significa hacer propios gran cantidad de conocimientos, habilidades, actitudes y valores necesarios para desenvolverse de manera autodirigida y ser productivo para la sociedad. Para lograr este fin, lo primero es socializar, es decir, conocer los códigos y conductas que permitan una relación adecuada con los miembros de la sociedad.

${ }^{1}$ GÓMEZ, H., Educación: la agenda hacia el siglo XXI, hacia un desarrollo humano., p.16. En lo referente a esta clasificación, consideramos que las funciones individuales son también sociales, y por otra parte, las funciones sociales enfatizan aspectos socio-económicos de carácter estructural. 
Dentro de la educación, también es importante transmitir la cultura, formada por el conjunto de saberes, costumbres y símbolos que la humanidad (y cada sociedad) han acumulado desde sus orígenes; representan los valores que conforman la conciencia colectiva (forma de ser, sentir, pensar y actuar de los miembros de un grupo), y con base en ellos, se estructura la identidad personal y grupal.

Quizá la más importante de las funciones de la educación es desarrollar la personalidad, que consiste en descubrir y alimentar las potencias individuales, y formar el carácter en valores éticos y estéticos.

El adecuado desempeño en el trabajo es, en gran medida, determinado por la educación. Es por eso que una de sus funciones sea la de formar para el trabajo, que incluye conocimientos y habilidades, que van tanto de lo abstracto a lo concreto, como de lo general a lo particular. La escuela debe cumplir con la enseñanza de lo abstracto y general, mientras que la empresa debe completar con lo concreto y particular. La escuela prepara para la empleabilidad y la empresa para el empleo.

La formación para la ciencia y la tecnología es otra de las funciones de la educación; dentro de ella se ha incluido la producción de conocimientos científicos y tecnológicos; sin embargo, si se analiza estrictamente, es el sistema de investigación quien se encarga de ello, aunque exista una estrecha relación entre dicho sistema y la educación. Por otro lado, la ciencia y la tecnología forman parte de la cultura y de la vida, por lo que la educación debe promover el que la población sea capaz de apoyarse en ellas.

En cuanto a la integración nacional, la educación permite entender el carácter y la historia de cada nación, "cada nación es su educación y viceversa " ${ }^{2}$. Reconocerse como nación es descubrir aquello que nos hace ser diferentes a los demás, que nos hace ser únicos y aportar algo al mundo. 
No puede llegarse a lo universal si primero no se profundiza en lo propio. Integrar la nación es hacer partícipes a todos sus ciudadanos de un código cultural común, de ciertas normas respetadas por todos que conforman la identidad nacional constituida por tres elementos: conciencia del pasado común (pasado); identificación del nosotros diferente de otros (presente); y un proyecto común (futuro).

La función en el crecimiento económico se entiende basada en el valor económico de la educación y puede analizarse desde tres perspectivas:

1) Los modelos clásicos y neoclásicos suponían que el crecimiento del producto se debe a tres variables: capital, trabajo (capital humano) y tecnología; éstas dos últimas son fuertemente influenciadas por la educación;

2) Existen seis nexos que la educación aporta al desarrollo económico: es indispensable para la innovación tecnológica; la productividad de la fuerza de trabajo depende principalmente de su nivel educativo; el espíritu empresarial es fruto del nivel y contenido de la educación recibida; el crecimiento de la racionalización social depende de la educación; la "ventaja competitiva de las naciones" depende de la eficacia de sus sistemas educativos; y la "inteligencia social", que es la capacidad de un país para adaptarse a un mundo que cambia, requiere una transformación en la forma de adquirir, usar y evaluar la información.

3) Se analizan las aportaciones que la educación hace al ingreso: a) Tasas de rentabilidad o de retorno a la inversión en educación, medidas en términos de costo-beneficio, en donde los beneficios representan el aumento de ingresos laborales producto de un año más de escolaridad, y los costos son todos los gastos por estudiar y los ingresos que sacrifica el estudiante por no trabajar (costo de oportunidad); b) proporción en el aumento del PIB (Producto Interno Bruto) que debe atribuirse a 
mejoras en la educación; y c) efecto de la escolaridad promedio sobre la expansión del producto $^{3}$.

La función de superación de la pobreza está íntimamente relacionada con el crecimiento económico antes mencionado, por ser el individuo quien recibe los frutos económicos de la educación. Además, el nivel y calidad de la educación influyen directamente en la productividad y el ingreso laboral. Como es bien sabido, la distribución del ingreso entre la población es desigual; sin embargo, una educación de calidad uniforme en todo el país, es generadora de igualdad de oportunidades. Finalmente, la educación de la mujer es un factor importante para la superación de la pobreza por las siguientes razones: genera menores tasas de fertilidad, menor mortalidad infantil y más educación para los hijos.

\section{CALIDAD DE LA EDUCACIÓN}

La educación es un derecho de toda persona. Hasta hace algunos años, la tendencia iba encaminada a la cantidad, pero no a los resultados de la misma: se enfatizaba más la cobertura y nivel de escolaridad, que lo oportuna que pudiera ser para quien la recibía. En la actualidad, esta tendencia ha cambiado: ahora la meta es brindar una educación de calidad que resulte significativa tanto para el desarrollo personal del alumno como el de la comunidad en la que se desenvuelve. De donde se desprende que el derecho a la educación incluya la calidad.

Para tener una visión más completa del término calidad, vale la pena analizarla desde su raíz etimológica: se deriva del latín qualitas, que significa cualidad. Desde entonces, calidad ha sido entendida desde diferentes perspectivas que pueden sintetizarse en tres: tradicional, modernizante y dialéctica ${ }^{4}$.

La connotación tradicional hace referencia a las propiedades que son derivadas de una esencia inmutable. La cualidad principal de la educación parte de la esencia de la persona, por ser el origen

${ }^{4}$ DELGADO SANTA, K., Evaluación y calidad de la educación., p.21. 
y fin de la acción educativa. La educabilidad es la propiedad en que la educación se basa; por lo tanto, una educación de calidad es aquella que fomenta el perfeccionamiento del ser humano.

Los valores desde el punto de vista modernizante son: el progreso, la civilización y lo moderno; en este sentido, la calidad se plantea en función de los resultados, dados por la eficacia en el proceso y la eficiencia en los medios. Así que la educación de calidad se alcanza cuando los medios conducen al logro de los objetivos y éstos son susceptibles de ser medidos, entonces los medios pueden ser modificados para el mejor logro de los fines.

Por su parte, la perspectiva dialéctica propone una transformación total de los medios, el proceso y los fines de la educación para promover un uso crítico del conocimiento acumulado y estimular la construcción, el descubrimiento, la capacidad problematizadora, la propia experiencia y la creatividad. Así se logra una verdadera relación teoría-práctica y la educación responde a las necesidades de la sociedad.

Así como la calidad de la educación es un término que puede ser abordado desde varias perspectivas, también es importante señalar que en ella participan los diferentes niveles de organización educativa: escolar, local, estatal, federal e internacional. Todos ellos son corresponsables en el logro de las metas educativas. Sin embargo, el término calidad adquiere diferentes matices de acuerdo a los factores que influyen en cada nivel. Sin pretender hacer un análisis exhaustivo del término, con este ensayo buscamos aclarar la relevancia del concepto en todos los sentidos.

\section{II.1. Calidad en el Proceso Enseñanza Aprendizaje (PEA)}

Entendemos como Proceso Enseñanza Aprendizaje, tanto al conjunto de relaciones que se dan entre los elementos que conforman el hecho educativo como tal -educando, educador, objetivos educativos, metodología, evaluación, recursos, comunidad educativa, familia...-, así como las formas de organización propias de la escuela. 
Algunos autores definen la calidad educativa como la relevancia del currículo y sus contenidos para los diferentes grupos étnicos y culturales; les preocupa que los estudiantes respondan a las necesidades de la comunidad en la que se desenvuelven. Siguiendo esta línea, señalan que los aprendizajes para la vida deben medirse no sólo en función del nivel de conocimiento teórico que posee el alumno, sino más bien en función a las habilidades con las que cuenta para poner dicho conocimiento en práctica y enfrentarse exitosamente a la vida.

De lo anterior puede concluirse que un concepto de calidad educativa debe contener qué y cuánto aprende el alumno, cuán relevante resulta este aprendizaje para que se desempeñe dentro de su comunidad y a su vez, cuánto lo capacita para producir los cambios que la sociedad necesita.

Para analizar este nivel (PEA), tomaremos como base el concepto de calidad educativa señalado por Víctor García Hoz en su libro Calidad de educación, trabajo y libertad, que brinda una concepción clara y completa: "La calidad es una determinación del ser. Se refiere no sólo a la materia, sino también y principalmente al ser mismo de las cosas, y, cuando se trata de personas, a sus caracteres psicológicos, espirituales" ${ }^{5}$. Para poder abordar el tema de calidad educativa desde esta visión, será necesario conocer el ser de la educación, cuya principal característica es que se trata de una actividad propia de la persona humana, de donde puede inferirse su complejidad otorgada por los múltiples factores que la influyen, principalmente el estar sujeta a la voluntad humana.

El autor agrega: "Se podría definir la calidad educativa como el modo de ser de la educación que reúne las características de integridad, coherencia y eficacia ${ }^{6}$.

A continuación presentamos los cuadros, que el autor incluye en su obra, para exponer cada uno de estos conceptos, seguidos de una pequeña explicación.

${ }^{5}$ GARCÍA HOZ, V., Calidad de educación, trabajo y libertad., p.2.

${ }^{6}$ Ibidem., p.3. 
Educación y naturaleza bumana (Educación esencial).

Perfección de todas las connotaciones de la naturaleza humana.

Intelectual

-Verdad

Moral

- Bien

Estética

-Belleza

Técnica

-Utilidad

-Necesidades materiales

Religiosa

-Lo absoluto

- Dios

Educación y vida (Educación existencial).

Respuesta a todas las exigencias de la vida.

Satisfacer todas las necesidades.

Biológicas

Capacidad física

\begin{tabular}{l|l} 
Psicológicas & $\begin{array}{l}\text { Sobriedad } \\
\text { Seguridad } \\
\text { Dignidad } \\
\text { Comunicación }\end{array}$
\end{tabular}

Todas las posibilidades de actividad.
Juego
Lucha
Estudio
Trabajo

En todos los ámbitos de la convivencia.

Familia -Amor y afirmación personal.

Profesión -Participación efectiva en la vida de la sociedad.

Amistad -Comunidad espontánea de sentimiento y servicio.

Fe -Comunidad religiosa.

Educación y persona bumana (Educación personalizada).

Desarrollo de todas las posibilidades de la persona.

Principio consistente de actividad.

Singularidad - Individualización

Autonomía - Libertad

Apertura - Inserción en la realidad ${ }^{1}$ Ibidem., p.7.

La calidad de la educación en cuanto a la integridad hace referencia al ser mismo de la educación e infiere que ésta sea completa, es decir, que incluya todos los aspectos de la naturaleza humana, que tienda siempre al desenvolvimiento de la 
persona y le permita alcanzar los valores que cada una de estas connotaciones le ofrece (Educación esencial).

Por otro lado, en la vida existen necesidades que deben ser satisfechas y posibilidades que deben hacerse realidad. La educación, requiere apoyar a la persona para satisfacer sus necesidades biológicas y psicológicas; capacitarla para desarrollar todas las posibilidades de actividad que la vida le ofrece y desenvolverse en todos los ámbitos de convivencia, en donde aprenderá los valores necesarios para poder relacionarse con otros (Educación existencial).

Finalmente, la educación debe desarrollar a cada persona como principio consistente de actividad, es decir, en su singularidad como ser individual diferente de otros; en su autonomía como principio de sus acciones y capaz de gobernarse a sí mismo; y en la apertura en sus tres niveles hacia las cosas, hacia los otros y hacia lo trascendente (Educación personalizada).

\section{CUADRO 2}

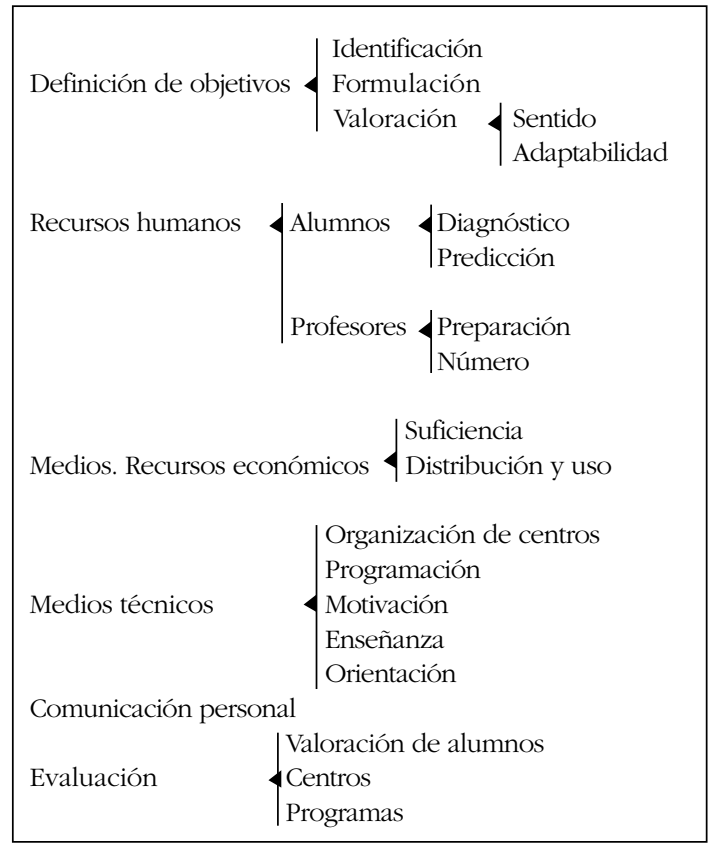


Se entiende por eficacia la indicación de que una actividad ha alcanzado el fin que persigue, opera en función de los objetivos que se han de alcanzar, utilizando los medios de los que se pueda disponer y finalmente evaluando si los resultados obtenidos son los que se esperaba.

El primer paso para la eficacia es la identificación, formulación y valoración de los objetivos, los cuales deben estar relacionados con la educación y los sujetos a quienes se dirige.

Después, se encuentran los recursos humanos, recursos materiales y medios técnicos. Dentro de los recursos humanos están los alumnos y los profesores, quienes llevan a cabo la acción educativa como tal; los materiales, se refieren a los recursos económicos que deben ser suficientes, bien distribuidos y utilizados adecuadamente; y los medios técnicos, que son la organización y métodos de las actividades educativas, tienen como fin que la labor tenga unidad y entidad propia. Para que la utilización, tanto de recursos como de medios, tenga éxito, es indispensable la comunicación personal entre los miembros de la comunidad educativa.

Finalmente, está el conocimiento de los resultados, es decir, la evaluación de los alumnos, centros y programas en función de los objetivos planteados.

CUADRO 3

Coherencia $^{3}$

Entre los fines generales de la educación exigidos por:

La naturaleza humana.

La vida y la comunidad humanas.

La persona singular ${ }^{4}$.

Entre los objetivos inmediatos y los fines generales de la educación.

De los distintos objetivos entre sí:

De aprendizaje específico.

De desarrollo personal.

Entre los objetivos y las actividades.

Entre la evaluación, los objetivos y los fines ${ }^{5}$.

Entre los distintos factores y ambientes educativos:

Técnicos.

Naturales.

Entre los padres, políticos y profesores.

De los profesores de un centro entre sí,

${ }^{3}$ Ibidem., p.13

${ }^{4}$ Véase el cuadro de integridad.

${ }^{5}$ Véase el cuadro de eficacia. 
La coherencia complementa la integridad y la eficacia dando el término de unidad, que se logra cuando los elementos que intervienen en la educación actúan de forma ordenada, de tal modo que la acción de cada uno no sólo no obstaculiza sino que contribuye a la acción de los demás y se ve reflejada en el conjunto.

\section{II.2. Calidad de los Sistemas Educativos}

Un problema actual al que se enfrenta la educación es la influencia ejercida por los modelos económicos que imperan en las sociedades en las que se desenvuelve: el acceso a la educación depende de la capacidad económica y no de los méritos de la persona. Vista así, la educación promueve y acrecienta la diferencia de clases.

Sin embargo, no resulta sencillo resolver las diferencias generadas por la educación. Para lograrlo, es necesario partir de que la calidad de los sistemas es dispar y desequilibrada de una región a otra. Ante este problema, las políticas educativas hoy tienden a eliminar las diferencias económicas y sociales promoviendo la cobertura y calidad educativa en todo el país; así, la educación se transforma en un factor de cohesión e instrumento de cambio.

Dichas políticas y los esfuerzos para mejorar la calidad deben orientarse para integrar a las personas pertenecientes a aquellos sectores más desfavorecidos, es decir, debe existir una discriminación positiva, con la cual se asignen mayores recursos a las personas más necesitadas, promoviendo 
el equilibrio en las posibilidades de acceso a la educación y la calidad de la misma entre los diferentes grupos sociales ${ }^{7}$.

En este sentido, es importante que las reformas educativas planteen objetivos bien definidos y que mantengan presente que el logro de éstos toma mucho tiempo; es necesario plantear metas claras a corto, mediano y largo plazo; asimismo, todas las políticas y programas que se implanten deben ser congruentes entre sí y orientarse a alcanzar los mismos objetivos. Sin embargo, lo más importante es que se mantenga el apoyo en los años subsecuentes; con este apoyo pueden alcanzarse los cambios gradual e integralmente, teniendo mayor garantía de éxito.

Otro de los problemas de la educación, íntimamente relacionado con la cobertura y la calidad, es la masificación y despersonalización de la misma: una educación de calidad no debe tender a educar por educar, sino atender en un primer momento las necesidades particulares de la comunidad y después responder a las necesidades específicas de la persona para que pueda descubrir sus propias capacidades; tal desarrollo se reinvierte en la sociedad al contar con personas capaces de generar ideas y transformar las realidades.

\footnotetext{
${ }^{7}$ En México, con la finalidad de mejorar la equidad en la distribución de oportunidades escolares y contrarrestar los efectos generados por no satisfacer los requisitos de calidad, se incluyeron en el capítulo III de la Ley General de Educación promulgada en 1993 medidas conocidas como preventivas, compensatorias y correctivas (o "remediales"), las cuales se pueden sintetizar en cinco puntos: a) asignar los recursos con fines compensatorios, es decir, ofrecer a los estratos sociales más desprotegidos los recursos e insumos de mayor calidad; b) diversificar la oferta educativa, atender las necesidades educativas de quienes no pueden integrarse al sistema educativo convencional o que han recibido atención deficiente; c) satisfacer la demanda por educación inicial y preescolar, para desarrollar en los niños habilidades que les permitan integrarse satisfactoriamente al sistema; d) mejorar los procesos educativos, con apoyos pedagógicos dirigidos a las poblaciones con necesidades específicas; y e) combatir los efectos de diversos obstáculos extraescolares que se interponen al aprendizaje. MUÑOZ IZQUIERDO, C., Calidad de la educación, políticas instrumentadas en diversos países para mejorarla., pp.114-117.
} 
La calidad educativa no puede equipararse, como se ha hecho en los últimos años, con escolaridad, principalmente por la inequidad en la calidad; por medio de ella se ha demostrado que los mismos años de estudio representan niveles de aprendizaje muy diferentes entre las escuelas efectivas y las escuelas marginadas.

Debido a ello, los sistemas educativos no pueden analizarse fuera del contexto en el que se desarrollan, pues éste le brinda ciertas particularidades. Por el contrario, es necesario analizar las relaciones que existen entre los diferentes elementos que conforman dicho contexto para comprender la influencia que ejercen en el sistema.

Si nos basamos en los elementos analizados anteriormente, puede inferirse que el desarrollo de un país se encuentra íntimamente relacionado con la calidad de la educación que se ofrece a la población ${ }^{8}$.

Para tener un acercamiento más preciso al término de calidad de educación en lo referente a los sistemas educativos, tomaremos como base las cinco variables que algunos autores han señalado como indicadores de calidad. Estas variables han sido aplicadas en el estudio de los diferentes sistemas educativos nacionales con el fin de conocer la interdependencia que existe entre la educación y el desarrollo socioeconómico. Los indicadores mencionados son los siguientes: eficiencia externa o pertinencia, eficiencia interna, efectividad, equidad y capacidad de gestión.

\footnotetext{
${ }^{8}$ Para el caso de México, la Constitución, en su artículo tercero, señala el derecho de todos los mexicanos de recibir educación y la obligación del gobierno de ofrecerla. Señala el mismo artículo que la educación impartida por el Estado tenderá a desarrollar armónicamente todas las facultades del ser humano y fomentará en él, a la vez, el amor a la Patria y la conciencia de la solidaridad internacional, en la independencia y la justicia. La fracción I del artículo señala el carácter laico de la educación y la fracción II añade tres criterios: la educación será democrática, nacional y contribuirá a una mejor convivencia humana.
} 
La primera variable es la eficiencia externa o pertinencia e indica "el grado en que el sistema educativo provee los conocimientos, aprendizajes, habilidades y destrezas requeridos en el mercado laboral (formal y no formal), así como los valores y actitudes que reclama una sociedad" ".

La eficiencia interna tiene dos formas de entenderse que pueden analizarse de manera independiente o interdependiente. De forma independiente, una tiene que ver con los flujos educativos dados por: la cobertura de ciertos grupos de la población, por el consumo ocasionado por la repetición y deserción, por el promedio de años que toma terminar un nivel educativo o por el paso de un nivel educativo a otro; la otra se relaciona con la forma en que se asignan y consumen los recursos: dinero, personas, cosas, tecnología, tiempo y organización escolar. De modo interdependiente, se entiende como la forma en que se utilizan los recursos (insumos) para generar, desde los niveles de conocimiento hasta los flujos educativos antes mencionados (productos) ${ }^{10}$.

La efectividad es el grado en que los alumnos adquieren los conocimientos, valores, actitudes, habilidades y destrezas que plantean los planes y programas de estudio del sistema educativo. Es importante señalar que la efectividad no implica la pertinencia, puede haber sistemas que cumplan con sus objetivos pero no cumplan con las demandas de la sociedad, o viceversa; de ahí que los objetivos deban plantearse conforme a las demandas sociales.

La equidad mide las diferencias que existen entre los distintos grupos de la población en cuanto a acceso, permanencia y egreso del sistema, en todos sus niveles y modalidades; así como la forma en que se ofrece el servicio dentro de cada uno.

9 PRAWDA, J., México educativo revisitado., p.138.

${ }^{10}$ Ibidem., p.139. 
La capacidad de gestión se manifiesta en dos niveles: a) la capacidad que tienen las autoridades de nivel superior para fijar políticas, estrategias y prioridades adecuadas al sistema, monitorear su ejecución, evaluar los resultados y asignar recursos a programas o grupos especiales; y b) la capacidad de las autoridades de los niveles inferiores de llevar a la práctica las políticas con acciones concretas, y administrar adecuadamente los recursos que le son asignados ${ }^{11}$.

Los indicadores que analizamos anteriormente se centran en el conocimiento de los procesos y resultados, pero conocerlos no implica mejorarlos. Un proceso de evaluación permite identificar los aciertos y errores; por sí solo no produce resultados, es necesario tomarlos como base para investigar acerca de los recursos y procesos que permitan mejorar la educación en cada caso específico ${ }^{12}$.

\section{II.3. Calidad de la Educación a Nivel Internacional}

Ante el proceso de globalización y la sociedad del conocimiento que imperan en el mundo, se ha hecho patente la necesidad de establecer a nivel internacional los estándares o parámetros con los cuales debe cumplir la educación de los diferentes países para poder ser considerada de calidad y uniforme en todas las naciones, respetando las culturas $\mathrm{y}$ diferencias individuales, y que permita el desarrollo equitativo a nivel mundial.

${ }^{11}$ Idem.

${ }^{12}$ En el caso de México, el Plan Nacional de Educación (PNE) 2001-2006, señala los tres grandes retos de la educación nacional: cobertura con equidad (especialmente en las regiones más desfavorecidas); calidad de los procesos educativos y los niveles de aprendizaje (comúnmente desiguales e inferiores a los estipulados; una educación de calidad desigual no puede considerarse equitativa); e integración y funcionamiento del sistema educativo (organización del sistema ante las condiciones cambiantes del entorno). Estos retos se expresan en tres principios fundamentales: educación para todos, educación de calidad y educación de vanguardia. SEP., Plan Nacional de Educación 2001-2006., p.16. 
A nivel internacional existen gran cantidad de instituciones interesadas en la educación como medio para lograr el desarrollo de las naciones, para fines de este ensayo hemos considerado a la UNESCO (Organización de las Naciones Unidas para la Educación, la Ciencia y la Cultura), por ser un organismo dependiente de la ONU (Organización de Naciones Unidas), principal organismo a nivel internacional. La UNESCO, está encargada específicamente de estudiar, analizar, evaluar, proponer, apoyar y promover el desarrollo en el ámbito educativo, y sus estudios tienen gran impacto en las políticas educativas de los diferentes países.

Se empezó a trabajar en educación de calidad a nivel internacional en los países en vías de desarrollo, una vez que se alcanzaron grandes logros relacionados con la universalización de la misma. Dichos países han instituido la calidad en sus programas educativos, trabajando en conjunto en los proyectos internacionales de la UNESCO, tales como el «Proyecto Principal de Educación para América Latina y el Caribe (1979)", cuyo objetivo principal es mejorar la calidad de la educación en los países de la región, y la "Declaración Mundial de Educación para Todos", firmada en Jomtien, Tailandia en 1990, a la cual se le da seguimiento en el Foro Mundial de la Educación de Dakar, Senegal 2000. Todos estos esfuerzos tienen como objetivo lograr un desarrollo equitativo de los países sustentado en la educación.

La "Declaración Mundial sobre Educación para Todos", expedida por la UNESCO en Jomtien, Tailandia en 1990, señala la importancia de cubrir las necesidades básicas de aprendizaje de todos los niños y jóvenes del mundo. Se entiende por necesidades básicas tanto las herramientas esenciales para el aprendizaje (lectura, escritura, expresión oral, cálculo y solución de problemas), como los contenidos básicos (conocimientos teóricos y prácticos, valores y actitudes) necesarios para que los seres humanos puedan sobrevivir, desarrollar plenamente sus capacidades, vivir y trabajar 
con dignidad, participar plenamente en el desarrollo, mejorar la calidad de su vida, tomar las decisiones fundamentales y continuar aprendiendo ${ }^{13}$.

Durante el "Foro Mundial de Educación de la UNESCO", en Dakar en 2000, se reafirma:

La visión de la Declaración de Educación para Todos (Jomtien, 1990), apoyada por la Declaración Universal de los Derechos Humanos y la Convención sobre los Derechos de los Niños, de que cada niño, adolescente y adulto, tiene derecho de beneficiarse de una educación que satisfaga sus necesidades básicas de educación en el mejor y más amplio sentido del término, una educación que incluya aprender a conocer, a hacer, a convivir y a ser. Se trata de una educación adaptada para aprovechar los talentos y el potencial de las personas y para desarrollar la personalidad de los educandos de modo que puedan mejorar su vida y transformar su sociedad ${ }^{14}$.

De lo anterior se infiere que una educación de calidad será aquella que incluya los cuatro pilares que la UNESCO propone: aprender a conocer, aprender a hacer, aprender a convivir $y$ aprender a ser. Los cuales explicaremos brevemente.

El primer pilar es el aprender a conocer lo cual va más allá de la simple adquisición de conocimientos; incluye las habilidades del saber, pudiéndose considerar a la vez un medio y un fin. "En cuanto medio, consiste para cada persona en aprender a comprender el mundo que le rodea, al menos suficientemente para vivir con dignidad, desarrollar sus capacidades profesionales y comunicarse con los demás. Como fin, su justificación es el placer de comprender, de conocer, de descubrir" ${ }^{15}$.

El aprender a conocer representa una forma de despertar la curiosidad intelectual, el análisis, la crítica y el juicio

\footnotetext{
${ }^{13}$ UNESCO., Declaración Mundial sobre Educación para Todos., en ww.unesco.org

${ }^{14}$ SEP-UNESCO., México en el Foro Mundial de Educación., p.35.

${ }^{15}$ DELORS, J., La educación encierra un tesoro., p. 92.
} 
que le permite al alumno dar respuesta a las preguntas que la vida le presente. Aprender a conocer implica primero un proceso de aprender a aprender por medio del cual, el alumno conozca su propio proceso de pensamiento y ejercite tanto la atención como la memoria. "La verdadera educación no sólo consiste en enseñar a pensar, sino también en aprender a pensar sobre lo que se piensa" (Fernando Savater). El fin de todo este proceso es que, con el paso del tiempo, el alumno encuentre en toda situación una oportunidad de aprendizaje.

De la mano con el aprender a conocer está el aprender a hacer, que se encuentra más íntimamente relacionado con la formación profesional de la persona. En otra época, la educación en este sentido era considerada como simple transmisión de la práctica, un tanto rutinaria. Sin embargo, en la actualidad, el aprender a hacer se ha vuelto una tarea de producción más intelectual y de diseño debido a que tanto las máquinas como el trabajo se han desmaterializado ${ }^{16}$.

Con el paso del tiempo, las exigencias en el campo laboral se han vuelto más específicas, requieren tanto una percepción técnica como profesional, necesitan de la interacción social y el desarrollo individual constante que permita dar respuestas prácticas e innovadoras a las necesidades cambiantes que presenta el medio.

Como es bien sabido, el hombre es un ser social y como tal, se ha desarrollado a lo largo de la historia de la humanidad; sin embargo la globalización hace latente, más que nunca, la necesidad de aprender a convivir, convertida en uno de los pilares antes mencionados.

El hombre siempre tiende a buscar su identidad, tanto personal como de grupo, tal tendencia genera un cierto rechazo hacia los demás propiciado también por la competencia natural que suele existir entre las personas y los grupos. En este sentido, el aprender a convivir se encamina a conocer

${ }^{16}$ Ibidem., p.95. 
y respetar las diferentes formas de ser y de pensar, lo cual no se logra únicamente con la organización del contacto y la comunicación entre los miembros del grupo y entre grupos. Es necesario tener como base un contexto de igualdad a partir del cual se formulen objetivos y proyectos comunes.

De lo anterior se desprende que la educación para la convivencia debe darse en dos orientaciones complementarias. En un primer nivel, el descubrimiento gradual del otro; en el segundo, y durante toda la vida, la participación en proyectos comunes ${ }^{17}$.

El primer nivel demanda un conocimiento de las semejanzas y las diferencias que existen entre las personas y entre los grupos, pero este conocimiento no es posible si no es parte de un autoconocimiento.

Por otro lado, la participación en proyectos comunes se relaciona directamente con el superar los hábitos individuales y valorar los puntos de convergencia por encima de los factores que separan; así, se da una nueva forma de identificación ${ }^{18}$.

El mundo actual, influenciado por la tecnología, parece tender a la despersonalización y masificación. Ante este problema, la UNESCO señala que uno de los pilares de la educación, el aprender a ser, debe encaminarse a desarrollar a las personas en su individualidad.

Desde sus inicios, este organismo ha mantenido firme un principio fundamental: "La educación debe contribuir al desarrollo global de la persona: cuerpo y mente, inteligencia, sensibilidad, sentido estético, responsabilidad individual, espiritualidad. Todos los seres humanos deben estar en condiciones, en particular gracias a la educación recibida en su juventud, de dotarse de un pensamiento autónomo y crítico y de elaborar un juicio propio, para determinar por sí 
mismos qué deben hacer ante las diferentes circunstancias de la vida" ${ }^{19}$.

El aprender a ser sintetiza en la persona todos los pilares de la educación: una vez que ha aprendido a conocer, ha aprendido a ponerlo en práctica por medio del aprender a hacer y a vivir en el mundo que lo rodea interactuando con los demás por medio del aprender a convivir y llegar al reto de hacer todo esto suyo, aprendiéndolo de manera personal: con el aprender a ser se forma la personalidad.

Los pilares antes mencionados no representan políticas educativas, son más bien aportaciones que la UNESCO hace y que pueden -y deben- ser adoptadas en todos los niveles para promover una educación más completa e integral y, por lo tanto, de mayor calidad.

En lo referente a las políticas internacionales, en el "Foro Mundial de Educación” de la UNESCO, en Dakar en 2000, se exige de todos los gobiernos y los demás asociados con la "Educación para todos", que colaboren para garantizar una educación básica de calidad, con independencia del género, riqueza, lugar, lengua u origen étnico. Se señalan ciertos requisitos indispensables para que un programa tenga éxito:

1) Alumnos sanos, bien alimentados y motivados.

2) Docentes bien formados y técnicas didácticas activas.

3) Locales adecuados y material didáctico.

4) Un plan de estudios que pueda enseñarse y aprender en una lengua local, y aproveche los conocimientos y la experiencia de profesores y alumnos.

5) Un entorno que no sólo fomente el aprendizaje sino que sea, además, agradable, atento a las cuestiones del género, sano y seguro.

6) Una definición clara y una evaluación precisa de los resultados esperados, entre ellos, los conocimientos, competencias, actitudes y valores.

${ }^{19}$ Idem. 
7) Un gobierno y una gestión participativos.

8) El respeto por la comunidad y la cultura local, y participación en ellas ${ }^{20}$.

Si analizamos cada uno de estos requisitos encontraremos grandes semejanzas con los parámetros de calidad señalados en los dos niveles anteriores; por lo tanto, si desde lo particular del hecho educativo se implementan y se van generalizando, es posible alcanzar la calidad, pero es necesario que cada nivel y el conjunto de factores que lo conforman, cumplan con el papel que les toca desempeñar dentro del proceso de calidad.

\section{CALIDAD DE LA EDUCACIÓN EN LA SOCIEDAD DEL CONOCIMIENTO}

Acompañándose de grandes cambios en el saber teórico y técnico, las tecnologías de la información y la comunicación propician el achicamiento del globo, pero también amplían el mundo del conocimiento.

Ante la globalización, los gobiernos tienen dos responsabilidades: al interior, preparar a su población para formar parte de esta aldea global, manteniendo la identidad nacional; y al exterior, insertar al país dentro de un nuevo orden económico, cultural y geopolítico mundial. El logro de este objetivo requiere la formación de una sociedad del conocimiento basada en la educación.

La sociedad del conocimiento se refiere a la tendencia actual de otorgar gran importancia a la ciencia y la tecnología, reconociéndolas como medio para desarrollar el saber necesario que permita a las naciones desenvolverse dentro del mundo globalizado. A continuación, revisaremos algunas de las principales características de la sociedad del conocimiento y sus demandas de calidad educativa.

${ }^{20}$ UNESCO., Foro Mundial de Educación., en www.unesco.org 
Dentro de la sociedad del conocimiento, las industrias más valoradas son aquellas que no dependen de recursos naturales, ni tampoco de mano de obra barata, son industrias cuyo factor de producción es la inteligencia y su producto es el conocimiento, por ejemplo, la biotecnología, las telecomunicaciones, la informática, la robótica, etcétera. Por lo tanto, el valor agregado lo da ahora la tecnología y el conocimiento ${ }^{21}$.

La educación de calidad, unida a esta demanda de conocimiento, enfatiza uno de los pilares de la educación señalados por la UNESCO: el aprender a aprender. Todos los niveles deben promover que los alumnos sean capaces de generar nuevos aprendizajes a partir de los que ya tienen y de las diferentes experiencias a las que se van enfrentando.

Otra de las características de la sociedad actual, que incide directamente en las demandas de la sociedad del conocimiento de actualización y generación de nuevos saberes, y además está estrechamente relacionada con la globalización, es la denominada aceleración de la historia: el tiempo cada vez es más corto y exige mayor velocidad de respuesta.

Dentro de una sociedad cambiante, todo debe cambiar a la misma velocidad. La sociedad del conocimiento demanda que las personas, instituciones y sistemas, se modifiquen y actualicen sus conocimientos para satisfacer las necesidades del medio que las rodea. Es necesario buscar siempre nuevas y mejores formas de hacer las cosas y dar solución a los problemas.

El PEA debe promover en los alumnos la creatividad y solución de problemas que les permitan pasar de un aprendizaje teórico a un aprendizaje práctico. Así, el conocimiento acumulado servirá de base para la innovación.

En lo referente a los sistemas educativos, éstos deben buscar la eficiencia externa o pertinencia, con la cual la

${ }^{21}$ GÓMEZ, H., op.cit., p.3. 
educación impartida responda a las necesidades de la sociedad. También es importante la capacidad de gestión mediante la cual los sistemas de administración se optimicen y permitan al sistema dar respuestas de forma más rápida.

Finalmente, la aceleración de la historia exige aprender a hacer, es decir, aplicar los conocimientos a la práctica y dar respuestas a las demandas de la sociedad.

Educar hoy es educar para el cambio. Sin embargo, el reto principal de la educación, en todos los niveles de organización, es identificar entre los aspectos esenciales que deben permanecer y los aspectos accidentales que pueden y deben cambiar para adecuarse a las necesidades de la sociedad $^{22}$.

Para que la educación sea de calidad, debe mantener los elementos señalados en cada uno de los niveles. PEA: integridad, eficacia $y$ coherencia; los sistemas educativos: eficiencia externa o pertinencia, eficiencia interna, efectividad, equidad y capacidad de gestión; y a nivel internacional: aprender a aprender, aprender a convivir, aprender a hacer y aprender a ser. Sin embargo, debe cambiar la forma en que se adquiere y utiliza la información.

En la actualidad, pueden identificarse dos tendencias claras: por un lado, la educación localista que propone la identidad nacional y valoración de lo propio, así como la referencia y pertinencia de la educación para las realidades locales. Por otro lado, la educación globalista promueve una educación en valores y saberes universalmente válidos; formar para la tolerancia que implica conocer y aceptar las diferencias ${ }^{23}$.

En este sentido, la educación no puede inclinarse por ninguno de los dos extremos; por el contrario, es necesario que se vuelva más completa y sea capaz de integrar ambas.

\footnotetext{
${ }^{22}$ Ibidem., p.44.

${ }^{23}$ Idem.
} 
Existen dos posturas en lo relativo a la circulación del conocimiento: la primera considera que la escuela debe seguir promoviendo la transmisión de conocimientos de forma organizada y sistemática; la segunda considera importante la contextualización y complementación del saber con los puntos de vista tanto individuales como de la comunidad, dando respuesta así a las necesidades individuales y sociales ${ }^{24}$.

La propuesta a esta demanda es que la transmisión de conocimientos -organizada y sistemática- incluya ejes transversales en los que se tomen en cuenta las necesidades propias y comunes.

Para la educación, la tecnología representa un arma de dos filos: abre nuevos espacios y crea herramientas útiles para todos sus niveles que, de ser bien empleadas, pueden apoyar en el logro de la calidad educativa y ampliar las distancias que existen entre los sectores de la población que tienen y los que no tienen acceso a ella. Para evitar este problema y obtener los mayores beneficios de la tecnología, es necesario que ésta llegue a toda la población, especialmente a quienes más lo necesitan. Evidentemente la tecnología debe estar acompañada de capacitación de maestros, para que ellos a su vez la puedan hacer llegar a los alumnos ${ }^{25}$.

Por otro lado, el cambio que la sociedad del conocimiento propone, se refleja en el ámbito laboral, al demandar de todos los oficios, mayores conocimientos técnicos y habilidades en el manejo de las nuevas tecnologías. El uso de la tecnología en el ámbito laboral genera la existencia de trabajadores supercalificados y vuelve innecesarios a los trabajadores no calificados. Ante esta situación, la educación no puede educar sólo a unos cuantos trabajadores supercalificados y dejar de lado al resto de la población. No sería ético, porque como se mencionó anteriormente, todos tenemos derecho a la educación, y a que ésta sea de calidad; 
la igualdad de oportunidades es indispensable para la integración nacional, el crecimiento económico y la superación de la pobreza. La integración de un país al mundo globalizado y a la sociedad del conocimiento no es posible si la mayoría de la población es improductiva y marginada.

Se puede afirmar entonces que, como señala Hernando Gómez, "(...) los países que se van integrando con éxito a la aldea global, son sociedades dirigidas por un claro proyecto nacional (...), son sociedades que han ejercido la inteligencia social, en la definición que le dio Stevan Deijer: "Habilidad organizada de un país para adaptarse a un mundo que cambia rápidamente, combinando la adquisición, evaluación y uso de información con actividades y operaciones planeadas en consecuencia” ${ }^{26}$.

La educación de calidad no debe inclinarse únicamente a promover en el alumno mayores conocimientos; debe impactar a la persona en su conjunto y educar en actitudes y valores que le permitan emplear adecuadamente esos conocimientos. Las actitudes son la forma en que la persona interactúa con el mundo y los valores son el eje que rige sus acciones.

Es necesario fomentar una actitud analítica y crítica que lleve a generar juicios de valor y desarrollar proyectos innovadores.

En una sociedad tan competitiva como en la que vivimos, es necesario poseer un fuerte deseo de superación que permita a los alumnos plantearse metas a corto, mediano y largo plazo; alcanzarlas implica esfuerzo, responsabilidad, amor al trabajo, entusiasmo y sobre todo tolerancia a la frustración, valores poco reconocidos en la sociedad actual en la que impera la «ley del mínimo esfuerzo".

El relativismo de los últimos años, ha dejado de lado el bien y la verdad, por ello la ética debe representar un eje primordial dentro de la educación de calidad.

${ }^{26}$ Citado por GÓMEZ, H., Ibidem., p.22.

REVISTA PANAMERICANA DE PEDAGOGÍA 


\section{CONCLUSIONES}

A modo de conclusión hemos elaborado un esquema que sintetiza, de forma gráfica, los diferentes elementos que intervienen en la calidad de la educación.

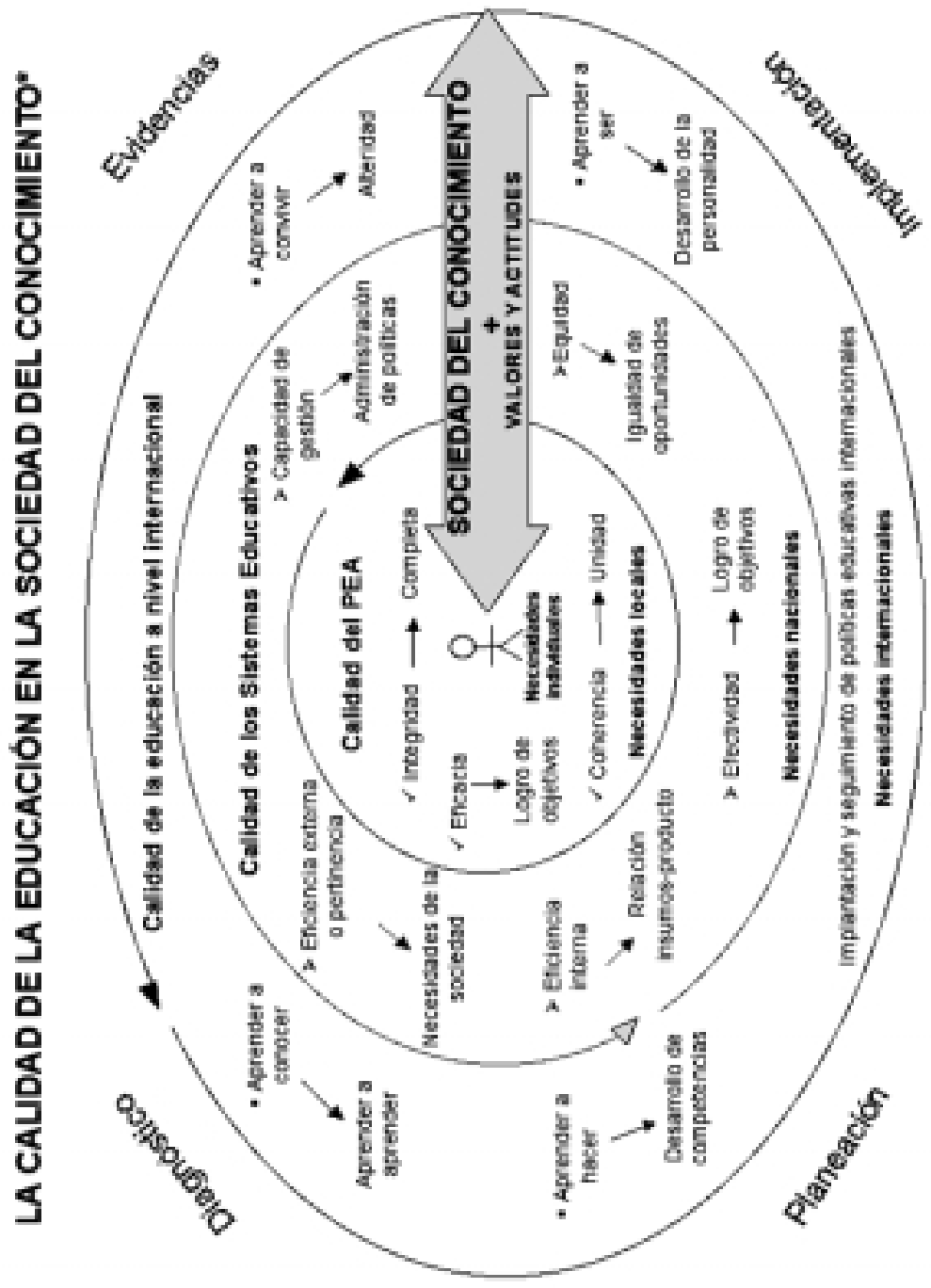


Del análisis realizado sobre la educación y sus funciones se puede concluir que ésta representa un medio para lograr el desarrollo sostenido (continuo) y sustentable (autofinanciado) de un país; por otro lado, el desarrollo educativo de una nación permea todo su progreso en conjunto. Resulta indispensable la educación para el despliegue social, cultural, tecnológico, económico y político.

La calidad educativa, en todos sus niveles de organización, es un proceso compuesto por varios pasos entre los que podemos incluir el diagnóstico de la situación actual, la planeación de nuevos planes y programas, la implementación de los mismos y la evaluación de los resultados. Sin embargo, la calidad es un proceso cíclico; cada evaluación representa también un nuevo diagnóstico. La calidad sugiere un proceso de mejora continua que a cada momento se revise y actualice.

Como puede observarse en el esquema, cada nivel presenta sus propias necesidades; la educación de calidad es aquella que satisface las necesidades de todos los niveles, permitiendo al alumno poseer un desarrollo personal e integrarse al contexto local, nacional e internacional. La educación debe adecuarse a estas necesidades y, conforme a ellas, plantear sus objetivos: una educación que resulte de calidad en un determinado momento y lugar, no implica que lo sea en cualquier momento y cualquier lugar. También debe tomarse en cuenta que las necesidades de una sociedad son cambiantes, por lo tanto, la educación debe estar en constante movimiento.

En el esquema podemos encontrar a la persona humana al centro, con lo que pretendemos representar que la calidad de la educación debe estar dirigida hacia las personas, de tal modo que las políticas y estrategias de todos los niveles tengan como fin la educación de personas de calidad.

Por otra parte, los maestros son quienes se encargan de hacer llegar la educación de calidad a los alumnos; por lo 
tanto, es indispensable que tengan la capacitación suficiente y la vocación necesaria para llevar a cabo la gran tarea de educar.

A lo largo de este ensayo pudimos notar que la calidad en el proceso educativo es un trabajo interdisciplinario, del cual todos los miembros de la sociedad (alumnos, padres de familia, empleadores, maestros....) somos beneficiarios y, por lo tanto, corresponsables. Una educación de calidad incluye no sólo la formal sino también la informal.

La calidad de la educación no es una tarea sencilla, requiere de constancia en todos los niveles: de los profesores dentro del aula; trabajo en equipo dentro de las instituciones educativas; continuidad en las políticas implementadas en los sistemas educativos y apoyo internacional.

Finalmente, en este ensayo puede concluirse que la sociedad del conocimiento, por sus características, está demandando que exista mayor relación entre los diferentes niveles de organización educativa para que la educación de calidad permita la inclusión de todos los individuos dentro de esta sociedad del conocimiento. El reto es mantener el equilibrio y dar su lugar a cada uno de los niveles; que cada uno cumpla con su misión, pero manteniendo una visión de conjunto. 
CÁZARES HERNÁNDEZ, L., et al., Técnicas actuales de investigación documental., México., Trillas., 1997.

DELGADO SANTA, K., Evaluación y calidad de la educación., Bogotá., Magisterio., 2000.

DELORS, J., et al., La educación encierra un tesoro., México., UNESCO., 1998., 302 p.

GARCÍA HOZ, V., Calidad de educación, trabajo y libertad., Madrid., Dossat., 1982.

GÓMEZ BUENDÍA, H., et al., Educación: La agenda del siglo XXI hacia un desarrollo humano., Colombia., PNUD/TM editores., 1998.

JimÉnEZ-OTTALENGO, R.; MORENO VAlle, L., Sociología de la educación., México., Trillas., 1997.

MUÑOZ IZQUIERDO, C., La calidad de la educación: políticas instrumentadas en diversos países para mejorarla., México., IFIE., 1998.

PRAWDA, J., FLORES, G., México educativo revisitado., México., Océano., 2001.

SCHMELKES, S., Hacia una mejor calidad de nuestras escuelas., México., SEP., 1992.

SCHMELKES, S., La calidad de la educación primaria., México., UNESCO., 1996.

SAKAIYA, T., Historia del futuro: La sociedad del conocimiento., Santiago., Editorial Ándres Bello., 1994.

SECRETARÍA DE EDUCACIÓN PÚBliCA., Programa Nacional de Educación 2001-2006., México., SEP., 2001. SECRETARÍA DE EDUCACIÓN PÚBliCA., Memoria del quehacer educativo 1995-2000., México., SEP., 2000.

SEP-UNESCO., México en el Foro Mundial del Educación Dakar 2000., México., SEP., 2000.

www.unesco.org 